\title{
Functional Connectivity as a Tool to Ascertain Language Localization in a Group of Children with Intractable Epilepsy
}

\section{Byron Bernal*, Magno R Guillen, Nolan Altman and Michael Duchowny}

Clinical Neuroscientist, Department of Radiology, Nicklaus Children's Health

System, Brain Institute, USA

*Corresponding Author: Byron Bernal, Clinical Neuroscientist, Department of Radiology, Nicklaus Children's Health System, Brain Institute, USA.
Received: December 31, 2020

Published: March 20, 2021

(C) All rights are reserved by Byron Bernal., et al.

\begin{abstract}
Introduction: We investigated the relationship between lateralization indices based on the of Brodmann areas (BA) connectivity and language fMRI paradigms in a group of children with intractable epilepsy.

Methodology: Intra-hemispheric ROI-to-ROI connectivity was assessed in 36 epileptic children (24 females) mean age 15.3 years, utilizing resting-state fMRI. Left vs right asymmetries were investigated between (1) BA pairs (pair-wise connectivity: PWC); (2) BA modules (global BA module connectivity: GBA); and (3) cerebral hemispheres (global hemispheric connectivity: GHC). Connectivity strength was graded in T scores (Z normalized values). Lateralization Index (LI) scores based on T values were obtained in all three assessments. Only LI with 2 SD above the mean were considered as significant lateralized pairs. Additionally, simple algebraic difference between left and right $\mathrm{T}$ values were obtained (TDIF).

Results: Connectivity-related LI of significant pairs (predictors) were regressed against Language-fMRI-based LIs. Sensitivity and specificity of significant predictors were found. Interactions with demographics, seizure features, and neuropsychological scores were also performed. Comparisons between T value-groups (per side and per BA) were performed utilizing double-sided T-tests. The null hypothesis was rejected for $\mathrm{P}<0.05$.

Conclusion: There was no statistical difference between left and right GHC strength $(\mathrm{P}=0.57)$, or by GBA-LI (LI = 0.0004). 6 BA pairs showed significant PWC-LI (> 0.424): BA9-BA19 (0.429); BA22-BA46 (-0.529); BA39-BA46 (0.679); BA39-BA42 (0.534); BA41-BA45 (-0.496); and BA42-BA46 (0.464). 5 BA pairs showed significant PWC-TDIF (> 3.32): BA9-BA47 (4.19); BA9-BA19 (3.81); BA21-BA45 (3.49); BA39-BA45 (3.90); and BA40-BA44 (-3.61). BA39-BA46 had a sensitivity of 80\% for language lateralization of both language domains.
\end{abstract}

Keywords: Epilepsy; Lateralization Index; Language; rs-fMRI; Functional Connectivity; fc-fMRI

\section{Introduction}

Pharmaco-resistant epilepsy is defined as the absence of seizure control despite several anti-seizure medications [1]. Excisional procedures have become the treatment of choice in patients with a defined seizure focus and achieve seizure freedom in a high proportion of patients [2]. Temporal or frontal resections in the dominant hemisphere may impact language areas [2,3]; rendering it critically important to lateralize language competence. 
Options to lateralize language in children are limited by poor cooperation and cognitive delays that compromise the accuracy of tasks involving naming, semantic discrimination and verbal fluency. Furthermore, procedures such as intracarotid amobarbital testing, intra-operative functional mapping, task-related functional MRI (fMRI) and magneto-encephalography (MEG) are challenging, even for children who are developmentally normal but too young to control movement or understand the task. Other children are cognitively limited by their underlying pathology or the adverse effects of medication.

Task-related fMRI has been adapted for non-cooperative patients utilizing passive paradigms to map visual, motor and auditory functions under light sedation $[4,5]$. Auditory activation utilizing speech as the stimulus in sleeping or sedated patients typically demonstrates clear lateralization of auditory areas that are similar to awake subjects who also may activate secondary auditory areas. This lateralization is likely related to asymmetric cortical specification that allows phonemic discrimination, a fundamental step for language comprehension. However, language lateralization through fMRI under sedation lacks of validation as corroborative methodologies are either invasive or difficult to implement in children. Asymmetries may arise not only from cortical specification differences, but also from connectivity, either structural or functional, and from cortical anatomical or vascular differences (e.g. [6-8]).

Resting-state-based functional connectivity magnetic resonance imaging (rsfc-fMRI) is a task-free procedure that provides insight into spontaneous brain oscillations. This endogenous activity is preserved even in sedated patients [9,10]. ROI-based rsfc-fMRI can quantify temporal correlations between specific brain regions, facilitating assessment of asymmetries of local connectivity strength and contralateral homotopic areas. Meta-analytic studies of coactivation derived from language fMRI have established functional connectivity of canonical and ancillary language areas [11-16].

In the present investigation, we sought to correlate asymmetries in brain connectivity between language areas and language lateralization scores derived from functional MRI (fMRI) mapping in a group of children with intractable partial epilepsy. We hypothesized that: (1) rsfc-fMRI-related brain connectivity of language is asymmetric; (2) connectivity-related lateralization scalars correlate with language lateralization indices; and (3) the main predic- tors of language lateralization are associated with Brodmann areas 44, 45, 21 and 22 (i.e. canonical Broca's and Wernicke's language areas). We assessed language brain connectivity strength and asymmetry at different levels: between the hemispheres (globally), at a functional modular level utilizing Brodmann's areas (BA), and at pairwise level (BA-to-BA).

\section{Methods}

Subjects

All patients were recruited from clinical cases referred to the radiology department of our hospital for a phase I MRI study as part of an evaluation for epilepsy surgery. Patients were recruited as part of a consecutive retrospective/prospective study, approved by the Western IRB. Patients within the prospective branch signed informed written consent.

Fifty-three patients were enrolled. All were right handed native English speakers, except for two left handers who were included with proven left sided language lateralization by fMRI. All underwent language mapping with fMRI utilizing a controlled task based on auditory description of objects, resting-state fMRI sequence, and 3D volumetric anatomical MRI. Seventeen patients were excluded from this group: 7 for motion of more than $2.0 \mathrm{~mm}$ either in translation or rotation present between any two contiguous time points either during language fMRI $(n=5)$ or during the resting state fMRI (rs-fMRI) ( $n=2), 5$ for overt motion during the anatomical series (data inspected by one of the authors -BB), as precise co-registration with templates may be affected, 4 with overt brain asymmetries or contour distortions that were judged to compromise co-registration with the normalization and segmentation atlas templates, and 1 because of parenchymal distortion from a tumor.

Thirty-six patients were studied (24 females) mean age 15.3, SD 3.2 years. Twenty-five had formal verbal IQ (V-IQ) assessment and 26 had global IQ scores (G-IQ) from the Wechsler Abbreviated Scales of Intelligence-Second Edition [17]. All but two were right handed, as determined by the writing hand. EEG and MRI, seizure type, onset and frequency were annotated for each case. Table 1 shows details of the demography.

\section{MRI technique}

All patients were scanned on a single MRI machine (Intera Philips Medical System 1.5 T MR Scanner, Philips Health Care, Nether- 


\begin{tabular}{|c|c|c|c|c|c|c|c|c|c|c|c|c|}
\hline \multirow[b]{2}{*}{ Case } & \multicolumn{3}{|c|}{ Demographics } & \multicolumn{2}{|c|}{ Neuropsych } & \multicolumn{2}{|c|}{ fMRI } & \multicolumn{5}{|c|}{ Seizures SPECS } \\
\hline & Gender & Handedness & Age & Verb IQ & Gen IQ & LI Exp & LI Rec & Sz Onset & Sz type & Sz Fq & EEG focus & MRI findings \\
\hline 1 & M & $\mathrm{R}$ & 17 & 91 & 86 & 0.9985 & 0.9849 & 2 & $\begin{array}{c}\text { Abs, head } \\
\text { drops }\end{array}$ & $1+/ w$ & Sharps W F3-T3 & NL \\
\hline 2 & $\mathrm{~F}$ & $\mathrm{R}$ & 16 & 104 & 92 & 1.0000 & 0.5417 & 8 & CPS & $1+/ y$ & Not found & R FCD \\
\hline 3 & $\mathrm{~F}$ & $\mathrm{R}$ & 12 & & & 1.0000 & 0.3083 & 1 & $\begin{array}{l}\text { Part Vis } \\
\text { (L) }\end{array}$ & $1+/ y$ & Spk R 0 & R FP gliosis \\
\hline 6 & M & $\mathrm{R}$ & 17 & & & 1.0000 & 0.9897 & 2 & GTC - Abs & $1+/ y$ & G Spk+W $2 \mathrm{~Hz}$ & NL \\
\hline 7 & $\mathrm{~F}$ & $\mathrm{R}$ & 18 & & & 0.5605 & 0.5828 & 6 & P S (L) & $1+/ m$ & Normal EEG & NL \\
\hline 8 & $\mathrm{~F}$ & $\mathrm{R}$ & 14 & 93 & 93 & 1.0000 & 1.0000 & 1 & CPS & $1+/ y$ & Sharps L F7-T3 & HS L \\
\hline 11 & $\mathrm{~F}$ & $\mathrm{R}$ & 15 & 91 & 100 & 1.0000 & 0.2886 & 2 & $\begin{array}{c}\text { CPS, Part } \\
\text { M }\end{array}$ & $1+/ m$ & Spk L T3-T5 & L FCD \\
\hline 13 & $\mathrm{~F}$ & $\mathrm{R}$ & 17 & & & -0.0480 & 0.5468 & 8 & $\begin{array}{c}\text { Abs; Part } \\
\text { Aut }\end{array}$ & $1+/ d$ & $\mathrm{Spk}+\mathrm{W}$ Bi F & NL \\
\hline 14 & $\mathrm{~F}$ & $\mathrm{R}$ & 19 & 89 & 86 & 1.0000 & 0.4093 & 1 & Part M (R) & $1+/ w$ & Sharps SW L P3 & $\begin{array}{c}\text { L FCD (Peri- } \\
\text { Syl) }\end{array}$ \\
\hline 15 & $\mathrm{~F}$ & $\mathrm{R}$ & 13 & 76 & 67 & 0.9986 & 1.0000 & 16 & Part M (L) & $1+/ d$ & Spk Cz-C3 & NL \\
\hline 16 & $\mathrm{~F}$ & $\mathrm{R}$ & 16 & 96 & 92 & 0.8519 & 0.5804 & 13 & $\begin{array}{c}\text { G Clonic } \\
(\mathrm{L}>\mathrm{R})\end{array}$ & $1+/ y$ & MultFoc Spk+W & NL \\
\hline 19 & $\mathrm{~F}$ & $\mathrm{R}$ & 12 & 112 & 117 & 0.6090 & 1.0000 & 2 & $\begin{array}{c}\text { GTC; Part } \\
\text { Psy (Deja- } \\
\text { Vu) }\end{array}$ & $1+/ d$ & Normal EEG & NL \\
\hline 20 & $\mathrm{M}$ & $\mathrm{R}$ & 21 & & & 1.0000 & 0.3155 & 14 & Part M (L) & $1+/ y$ & Spk T4 & Bulb L hipp \\
\hline 23 & M & $\mathrm{R}$ & 17 & & & 0.4684 & 0.5356 & 9 & CPS & $1+/ m$ & $\begin{array}{c}\text { R T } \\
\text { Spk+PolySpk+W }\end{array}$ & R T FCD \\
\hline 24 & $\mathrm{M}$ & $\mathrm{R}$ & 12 & & & 1.0000 & 0.6993 & 1 & Part M & $1+/ w$ & R F Spk & $\mathrm{NL}$ \\
\hline 25 & $\mathrm{~F}$ & $\mathrm{R}$ & 16 & & & 0.8595 & 0.6711 & 15 & $\mid \begin{array}{c}\text { Part Psy } \\
\text { (Jamis-vu) }\end{array}$ & $1+/ d$ & Sharps T6 & NL \\
\hline 26 & M & $\mathrm{R}$ & 17 & & & 0.9250 & 0.9794 & 1 & Abs; TCG & $1+/ m$ & $\begin{array}{c}\text { Bi-F G Spk+W } \\
3-4 \mathrm{~Hz} \\
\end{array}$ & NL \\
\hline 27 & M & $\mathrm{R}$ & 13 & 103 & 99 & 0.5802 & 0.9429 & 2 & $\begin{array}{c}\text { Part S } \\
\text { (Dizz); } \\
\text { CPS } \\
\end{array}$ & $1+/ m$ & R Sharps F8-T4 & R T FCD \\
\hline 29 & $\mathrm{~F}$ & $\mathrm{R}$ & 17 & 96 & 96 & 0.9779 & 0.0188 & 6 & $\begin{array}{l}\text { Part Aut; } \\
\text { Part S (L) }\end{array}$ & $1+/ m$ & $\begin{array}{c}\text { R T } \\
\text { Spk+PolySpk }\end{array}$ & $\begin{array}{c}\mathrm{L} \mathrm{T} \\
\text { Cavernoma }\end{array}$ \\
\hline 30 & $\mathrm{~F}$ & $\mathrm{R}$ & 16 & 99 & 93 & 0.4274 & 0.8311 & 12 & CPS & $1+/ m$ & $\begin{array}{c}\text { Sharps T } 5 \text { (> } \\
\text { T6) }\end{array}$ & L FCD \\
\hline 31 & $\mathrm{~F}$ & $\mathrm{R}$ & 17 & 105 & 109 & 0.0963 & 0.7384 & 1 & $\begin{array}{c}\text { Part Psy } \\
\text { (Déjà vu); } \\
\text { CPS }\end{array}$ & $1+/ d$ & Fast Ryt T3-T5 & Bulb L hipp \\
\hline 52 & $\mathrm{~F}$ & $\mathrm{R}$ & 15 & 106 & 101 & 1.0000 & 1.0000 & 10 & CPS & $1+/ w$ & $\begin{array}{c}\text { Sharps T4, T6, } \\
02 \\
\end{array}$ & $\begin{array}{c}\text { R OP messial } \\
\text { FCD }\end{array}$ \\
\hline 54 & $\mathrm{~F}$ & $\mathrm{R}$ & 19 & 77 & 103 & 1.0000 & 0.6539 & 12 & Part Vis & $1+/ w$ & $\begin{array}{c}\text { Delta Ryt P08, } \\
02,0 z\end{array}$ & $\begin{array}{c}\text { R O } \\
\text { heteroTopia }\end{array}$ \\
\hline
\end{tabular}




\begin{tabular}{|c|c|c|c|c|c|c|c|c|c|c|c|c|}
\hline 55 & $\mathrm{~F}$ & $\mathrm{R}$ & 10 & 91 & 93 & 1.0000 & 0.8127 & 10 & Part S (R) & $1+/ d$ & Spk C3 & $\begin{array}{c}\text { L Cent } \\
\text { Ganglio- } \\
\text { glioma }\end{array}$ \\
\hline 56 & $\mathrm{~F}$ & $\mathrm{R}$ & 16 & 87 & 82 & 0.1037 & 0.6210 & 1 & CPS & $1+/ m$ & Spk T3-F7 & L T HS; FCD \\
\hline 57 & $\mathrm{~F}$ & $\mathrm{R}$ & 16 & 108 & 100 & 0.0171 & 0.1295 & 10 & $\begin{array}{l}\text { Part Psy; } \\
\text { CPS }\end{array}$ & $1+/ w$ & Spk T6 & $\begin{array}{c}\mathrm{R} \\
\text { Hemisphere } \\
\text { Atrophy }\end{array}$ \\
\hline 59 & $\mathrm{~F}$ & $\mathrm{R}$ & 23 & & 70 & 0.8647 & 0.5118 & 18 & CPS & $1+/ w$ & Spk+W T4 & $\begin{array}{c}\mathrm{R} \mathrm{T} \\
\text { Encephalo- } \\
\text { malacia; R HS }\end{array}$ \\
\hline 60 & $\mathrm{~F}$ & $\mathrm{R}$ & 10 & 76 & 79 & 0.3694 & 0.9087 & 5 & CPS & $1+/ w$ & $\begin{array}{c}\text { Spk T3-T5; Gen } \\
\text { Spk+W }\end{array}$ & L T HS \\
\hline 62 & M & $\mathrm{R}$ & 12 & 90 & 78 & 0.6467 & 0.9952 & 11 & Part Aut & $1+/ w$ & Delta Ryt T4-F8 & R T HS \\
\hline 65 & $\mathrm{~F}$ & $\mathrm{R}$ & 10 & 118 & 127 & 0.9996 & 1.0000 & 6 & Part M (L) & $1+/ d$ & Spk F4-F6 & R FP FCD \\
\hline 66 & M & $\mathrm{R}$ & 18 & 59 & 75 & 0.5356 & 0.9575 & 8 & $\begin{array}{c}\text { CPS; Part } \\
\text { Psy }\end{array}$ & $1+/ d$ & L T Spk & $\begin{array}{c}\text { L T } \\
\text { Ganglio- } \\
\text { glioma }\end{array}$ \\
\hline 67 & $\mathrm{M}$ & $\mathrm{R}$ & 13 & 102 & 92 & 1.0000 & 0.8748 & 9 & Part M (L) & $1+/ \mathrm{m}$ & Sharps Bi-front & R P Tumor \\
\hline 69 & M & $\mathrm{R}$ & 18 & 75 & 80 & -0.3146 & 0.0367 & 9 & $\begin{array}{l}\text { Part Vis; } \\
\text { CPS }\end{array}$ & $1+/ d$ & R > L TO Sharps & NL \\
\hline 70 & $\mathrm{~F}$ & $\mathrm{~L}$ & 8 & 99 & 107 & 1.0000 & 0.1851 & 7 & $\begin{array}{c}\text { Part Psy; } \\
\text { Part M (R) }\end{array}$ & $1+/ d$ & Sharps T3 & NL \\
\hline 71 & M & $\mathrm{R}$ & 15 & 112 & 111 & 1.0000 & 0.6331 & 1 & $\begin{array}{l}\text { Part Vis } \\
\text { (L); CPS }\end{array}$ & $1+/ m$ & $\begin{array}{c}\text { Spk+W 1-3 Hz } \\
\text { 02-T6 }\end{array}$ & $\begin{array}{c}\text { R OP Sturge } \\
\text { Weber }\end{array}$ \\
\hline 72 & $\mathrm{~F}$ & $\mathrm{~L}$ & 15 & & & 1.0000 & 1.0000 & 15 & $\begin{array}{l}\text { Part Vis } \\
\text { (L); GTC }\end{array}$ & $1+/ y$ & Normal EEG & \begin{tabular}{|c|} 
L O \\
Developmen- \\
tal Tumor \\
\end{tabular} \\
\hline & & Mean & 15.29 & 94.20 & 93.38 & & & & & & & \\
\hline & & SD & 3.22 & 13.89 & 14.36 & & & & & & & \\
\hline
\end{tabular}

Table 1: Detailed demographics, neuropsych scores, language scores and clinical history.

Abs: Absence; Aud: Auditory; Aut: Autonomic; Bulb: Bulbous; CPS: Complex Partial Seizure; d: Day ; Exp: expressive language; F: Frontal; FCT: Focal cortical dysplasia; Fq: frequency; Gen IQ: General IQ; GTC: Generalized Tonic Clonic; Hip: Hippocampus; HS: Hippocampal sclerosis; LI: Lateralization Index ; m: Month; M: Motor; NL: Non lesional; O: Occipital; P: Parietal; Part: Simple Partial Seizure ; Psy: Psychic; Rec: receptive language; S: Sensory; Spk: Spike; Sz: Seizure; T:Temporal; TCG: Tonic Clonic generalized; Verb IQ: Verbal IQ ; Vis: Visual; w: Week ;W: Slow wave; $y$ : Year; 1+ /: Several per. Blank spaces correspond to unavailable data.

*: Edinburgh Handedness Inventory.

**: Wechsler Abbreviated Scale.

lands). Anatomical T1-weighted MRI 3D-volume was acquired in the axial plane with a FOV of $240 \times 240 \mathrm{~mm}$, voxel size $1 \times 1 \times 1$ $\mathrm{mm}, 120$ - 176 slices in one slab, slice thickness of $1 \mathrm{~mm}$, and the following acquisition settings: TR: $25 \mathrm{~ms}$, TE: $3.8 \mathrm{~ms}$ and flip angle of 30 degrees. The first BOLD-sensitive echo-planar sequence was acquired to determine language lateralization. This fMRI sequence 
consisted of 150 time points acquired in 5 minutes of scan time with the following parameters: FOV $240 \mathrm{~mm}$, matrix 64 x 64 voxels (voxel size $3.75 \times 3.75 \times 6.0 \mathrm{~mm}$ ), $14-21$ axial interleaved slices with no gap, TR: $2000 \mathrm{~ms}$, TE: $45 \mathrm{~ms}$, flip angle 90 degrees, standard shim mode. The second BOLD-sensitive sequence, aimed to obtain the rs-fMRI, consisted of 200 time-points (scan time 6:40 minutes), keeping the rest of settings identical to the first fMRI.

\section{Paradigms}

The language fMRI consisted of a block design auditory description task (ADT). In this paradigm, the subject is presented with 30 seconds of statements in English (task epoch). Statements may be either true or false. The patient is instructed to press a button whenever he/she judges the statement to be true. 18 of 35 total statements are true. As a control, each patient is presented with 30 seconds of gibberish composed of the probe statements played backwards (rest epoch). During the rest epoch, the gibberish is split into pseudo-phrases some of which are followed by a beep. The patient is instructed to press the button each time a beep is heard. The number of beeps during the control block matches the number of true statements in the task to compensate the button pushing action. Auditory stimuli were provided using MRI compatible headphones fed by a plastic hose from an fMRI compatible ceramic speaker located by the magnet (fMRI Consulting, Miami, USA); patient responses were given by pressing a button connected to the operator's room via fiber optics. For the rs-fMRI session all patients were instructed to keep their eyes closed and focus their attention on the breath, trying to feel the air flow in the nostrils.

\section{Pre-processing}

Due to the nature of this research, extreme care was taken at all processing steps to verify the " $\mathrm{x}$-axis" true orientation of all series. All prospective case series were labeled with an in-house marker placed to the right side of the headphones. This marker was visible in T1 and EPI sequences as it has a concentric array of small tubes filled with water and vitamin E oil. For the rest of the cases the true right-to-left orientation of the anatomical volume was verified via vis-a-vis comparison with the clinical images available in PACS. Minor asymmetries of the brain and skull, including the occipital torque, ventricular size, nasal septum and differences on the petrous bone axial location were utilized to verify the orientation of all the fMRI volumes.
After verification of proper orientation, each anatomical volume was co-registered with the Montreal Neurological Institute (MNI)152 standard space template. Co-registration was performed automatically using FMRIB's Linear Image Registration Tool (FLIRT version 5.5). Each co-registration result was visually validated.

\section{fMRI}

fMRI series with motion of more than $2.0 \mathrm{~mm}$ detected automatically by the preprocessing software, were discarded.

Task-related fMRI data sets were re-aligned, normalized, and spatially smoothed utilizing a Gaussian kernel of full width at half maximum of $7 \mathrm{~mm}$ using fMRI Expert Analysis Tool (FEAT) version 5.98 from FSL 4.1 .9 (http://www.fmrib.ox.ac.uk/). The dataset was initially co-registered to the patient's anatomical volume and transformed into the MNI-152 standard space template similar to the method in the prior co-registration. Each co-registration result was visually validated.

\section{Rs-FMRI}

Pre-processing was performed utilizing SMP12 (www.fil.ion. ucl.ac.uk/spm/) and MATLAB 8.6, R2015b. Data was re-aligned, normalized, and spatially smoothed utilizing a Gaussian kernel of full width at half maximum of $7 \mathrm{~mm}$. Anatomical volumes were segmented into gray and white matter, and CSF areas and the resulting masks eroded in one voxel dimension to minimize partial volume effects. The temporal time series characterizing the estimated subject motion ( 3 translations, 3 rotations, and 6 parameters representing their first-order temporal derivatives); the BOLD effect of the white matter mask (3 principal component parameters); and the CSF (3 principal component parameters) were used as temporal covariates and removed from the BOLD functional data using linear regression methods. The resulting residual BOLD time series were then band-pass filtered $(0.008<\mathrm{f}<0.09)$. The rs-fMRI was first co-registered to the structural $\mathrm{T} 1$ anatomical image and then to MNI-152 standard space with same method described above.

\section{Processing \\ fMRI processing}

The task-related fMRI post-processing was performed using fMRI Expert Analysis Tool (FEAT) version 5.98 from FSL 4.1.9 [18]. The block design model consisted of ten 30-second-epoch blocks starting in the off-condition and convoluted with a double gamma 
hemodynamic response function. Activation maps were generated by FEAT using the FMRIB's Improved Linear Model (FILM) in a univariate analysis fitting the model to each voxel's time-course separately. FILM allows an accurate nonparametric estimation of time series autocorrelation to pre-whiten each voxel's time series; Statistical results were corrected for multiple comparisons using Gaussian Random Field theory $(\mathrm{Z}>2.3$, clusterwise $\mathrm{p}<0.01$, onetailed). Each activation map was co-registered using FLIRT to the previously spatial-normalized anatomical images.

\section{Language lateralization index}

For each subject, independent expressive and receptive LIs were obtained by comparing domain-specific ROIs created with the following method. We initially obtained expressive and receptive ROIs that could contain all the subject's activations. For this purpose, we spatially summed all the spatially normalized voxels of activation per domain and accepted any voxel within two standard deviations based on the frequency of voxel activation. The largest ROI between left vs right was then chosen and a mirror ROI was obtained by flipping it along the sagittal midline. The process was carried out in the Multi-Image Analysis GUI (MANGO) developed by the Research Imaging Institute of the University of Texas (http:// rii.uthscsa.edu/mango/). The two ROIs (left and right) corresponding to the expressive language areas included Brodmann's area (BA) 44 and BA45, and partially BA6, BA9, BA46 and BA47. The two ROIs (left and right) corresponding to receptive language included BA21, BA22, BA37, BA39, BA40, and also BA19, BA41, BA42. See figure 1. After binarization of these ROIs, a script was created to multiply the subject's activation maps with each of the four created ROIs allowing to count the number of voxels involved per side and per language domain. Lateralization indices (LI) were calculated independently for expressive and receptive language from the taskrelated fMRI for each patient using the equation:

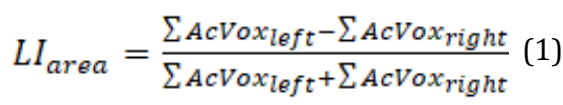

In which $\mathrm{AcVox}_{\text {left }}$ and $\mathrm{AcVox}_{\text {right }}$ represent the total count of active voxels in the left and right hemispheres respectively. Positive values indicate left lateralization, while negative values mean rightward lateralization. Usually, in the clinical field, values below -0.2 are considered rightward, values over 0.2 leftward; values in- between are not considered significantly lateralized. The same principles of the LI formula described in equation (1) were uti- lized to compute LI of connectivity strength. The numerator was represented by the difference (left minus right) of the connectivity strength given in $\mathrm{T}$ absolute values and the denominator as their sum (see equation 2). There are two reasons to utilize absolute values- to keep the range of results between -1 and 1, and to avoid denominators of value 0 . Further explanation is given in Limitations and Future Directions.

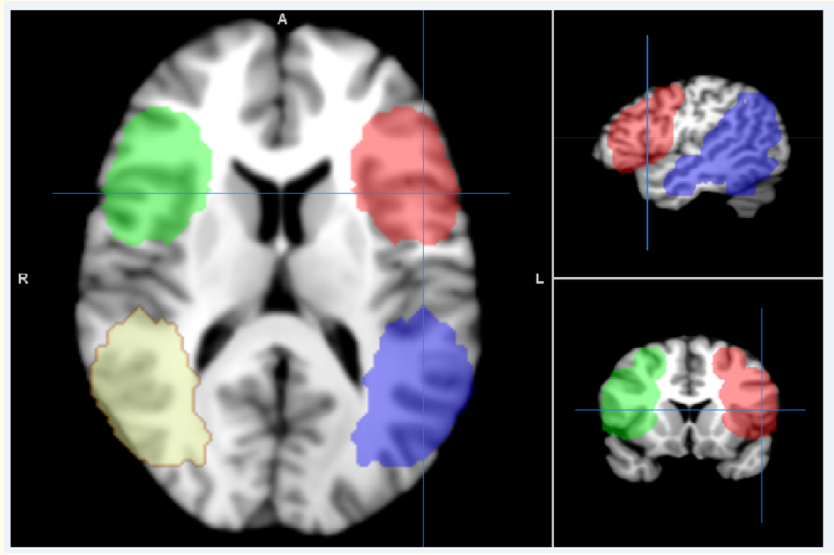

Figure 1: ROIs used to assess Lateralization Index for Language Areas: left expressive region (red), left receptive region (blue), right expressive region (green) and right receptive region (yellow).

Rs-fmri processing

First level analysis

Functional Connectivity was performed utilizing the MATLAB toolbox "CONN" [19] version 15.g available at www.nitrc.org/projects/conn. We executed CONN within a MatLab 8.6, R2015b. Seeding ROI sources were derived from the atlas of Brodmann's areas (BA) provided by SPM12. Intra-hemispheric ROI to ROI connectivity between functional areas was assessed. (Interhemispheric connectivity was not considered in this study). Based on prior published meta-analyses of co-activations present in language tasks [11-13,15,16]. 32 BA were selected --16 per hemisphere. These included: BA6, premotor; BA7, dorso-parietal; BA9, pre-frontal; BA19, secondary visual; BA21 and BA22, Wernicke's area; BA37, fusiform gyrus; BA38, temporal pole; BA39, angular gyrus; BA40, supramarginal gyrus; BA41 and BA42, primary auditory area; BA44 and BA45, Broca's area; BA46, prefrontal; and BA47, pars or- 
bitalis of the inferior frontal gyrus (IFG). With these areas we have at our disposal 120 possible intra-hemispheric pairs. For each patient, CONN generated a BA-based connectivity strength reports in terms of beta, T, p-uncorrected and p-FDR corrected T values. Statistically significant connectivity was accepted for those pairs with p-corrected values below 0.01. From these tables, two independent reports were generated referring to the left and right intra-hemispheric connectivity. The inter-hemispheric connectivity was not processed.

\section{Interactions and statistical methods}

All patient population was analyzed with CONN using "all subject" effects per target BA obtained (between-sources contrast 1,0), using the following settings Within-subject effects of the betweensources contrasts correspond to multivariate/repeated-measures analyses of the selected effects modeled using a general linear model. The output is a within-subject linear combination of effects specified by the "between-conditions" and "between-sources" contrasts, applied to the first-level connectivity-measure matrix (the ROI-to-ROI analyses). A table automatically generated was obtained. This table reported the strength of intra-hemispheric connectivity with the following columns: Source BA, Target ROIs, T value, P-uncorrected and P-corrected value. An additional column was added computing the Lateralization Index (LI) per BA-pair. For this calculation, all $\mathrm{T}$ values were corrected to absolute values, since negative correlation are expressed as negative $\mathrm{T}$.

As our approach does not differentiate directionality, a further reduction of the data was accomplished by discarding duplicated data. Thus, connectivity between Brodmann areas A and B has the same value than connectivity between $B$ and A. For clarity, we will name the connectivity relationship from lower to higher BA. Thus, the connectivity between BA44 and BA21 is designated BA21BA44.

Statistically significant ROI-to-ROI connectivity of this group was accepted with a threshold fixed at $\mathrm{p}<0.05$ (two-sided) and corrected for multiple comparisons with the False Discovery Rate (FDR) technique. From the report provided by CONN, 2 separated tables (right and left intra-hemispheric connectivity) were obtained.

The following parameters were computed for each ROI pair: T value, as well as uncorrected and corrected (FDR) p-values. Only pairs with $\mathrm{p}<0.05$ FDR corrected were accepted, which resulted in a minimum $\mathrm{T}$ value of 2.3. Pairs were sorted by $\mathrm{T}$ values obtained from the left hemisphere. Values for the right-hemisphere homotopic pairs were identified and annotated for each pair. Post-hoc analysis was performed in cases in which the counterpart did not reach threshold.

To ascertain connectivity asymmetries, similar to the calculations performed for the task-related fMRI, LI per BA-pair/side based on $T$ values were calculated, utilizing the formula:

$$
\mathrm{LI}=\frac{|T l|-\left|\mathrm{T}_{\mathrm{r}}\right|}{|T 1|+\left|\mathrm{T}_{\mathrm{r}}\right|}
$$

Where $|\mathrm{Tl}|$ is the absolute $\mathrm{T}$ value of the left BA connectivity, and $\left|\mathrm{T}_{\mathrm{r}}\right|$ is the absolute $\mathrm{T}$ value of the right BA connectivity. Values below -0.2 are typically considered rightward and values over 0.2 leftward; values in- between are not considered significantly lateralized, but we accepted values exceeding 2SD from the LI distribution of the sample as lateralized. The utilization of absolute values of $\mathrm{T}$ permitted a focus on strength of connection. Anti-correlations indicated by negative $\mathrm{T}$ values are related to inhibitory input and addressed separately.

\section{Comparisons and correlations}

Several parametric variables were obtained from the T results. Three related to connectivity strength, and three related to asymmetry of connectivity strength. Connectivity methods of analysis and their variables are graphically represented in figure 2 . Connectivity strength variables were: Pair-wise connectivity (PWC), defined as the non-directional connectivity strength (in $\mathrm{T}$ value) of a given BA pair; Global Brodmann's Area connectivity (GBAC) as the average of the strength of all connections (within the selected BA areas) belonging to a specific BA module; and Global Hemispheric connectivity (GHC) as the average of all connection's strength among the selected BAs. Asymmetry variables consisted of lateralization indices derived from the T values of each PWC (PWC-LI), and the GBA and GHC asymmetries (GBA-A and GHC-A, respectively). For the LI we utilized the equation (2): GBA-A and GHC-A asymmetries were assessed as mean differences between the left and right hemispheres and were tested for statistical significance utilizing two-tailed $\mathrm{T}$ test, with a threshold set at $\mathrm{p}<0.05$. GBA-A and GHC-A were also analyzed averaging the sum of the PWC-LI per BA and per hemisphere respectively. 

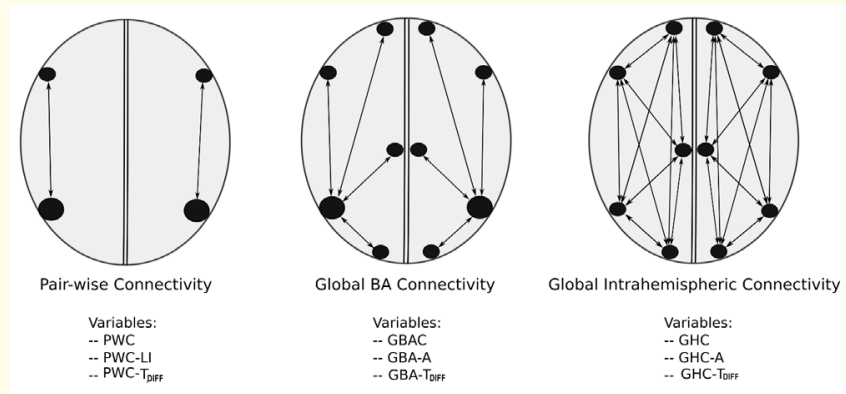

Figure 2: Different types of connectivity analyses. Pairwise: connectivity (PWC) strength, lateralization index (PWC-LI) and connectivity T value difference (PWC-TDIF) among Brodmann areas; Global Brodmann Area: connectivity strength (GBAC), connectivity asymmetry (GBA-CA) and connectivity T value difference (GBA-TDIF) among Brodmann areas; Global Intra hemispheric: connectivity strength (GHC), connectivity asymmetry (GHC-A) and connectivity T value difference (GHC-TDIF) among Brodmann areas.

Selection of significant pairs. The PWC-LI obtained in all comparisons were averaged and the group SD found. Those PWC-LI exceeding 2 SD above the mean were accepted as statistically significant and selected to be used as possible predictors. For this purpose, all PWC-LI values, each side apart, were considered as the universe of the sample. No directionality in the connectivity was considered, thus pair BA6-BA9 is the same as BA9-BA6. In addition to lateralization index based on absolute values, the asymmetry between BA pairs were assessed by a simple algebraic difference between sides by subtracting the Right $\mathrm{T}$ value from the Left $\mathrm{T}$ value $\left(\mathrm{T}_{\mathrm{DIF}}\right)$. The higher the $\mathrm{T}_{\mathrm{DIF}}$ value, the more left lateralized the connectivity is. Statistical significance was defined as values above and below 2 SD from the mean of the distribution.

For each patient, the PWC-LI and PWC-T ${ }_{\text {DIF }}$ values of the significant pairs were found. The list of these values in the entire group was regressed against their corresponding expressive and receptive LIs. No regression was performed with GBAC of BA9 as we did not find any significant correlation with its 3 related pairs (BA9BA19 from GBA-LI and BA9-BA19 and BA9-BA47) from GBA-TDIF.
In the next step, to further explore the clinical suitability of these scores to predict language lateralization, the significant PWC-LIs were regressed at subject level against the patient's language LIs for both domains (expressive and receptive). In this way we wanted to assess the sensitivity and specificity of the predictors as well as the error type I and II associated to them.

The sensitivity (Se) and Specificity ( $\mathrm{Sp}$ ) were assessed using the following standard equations:

$$
\begin{aligned}
& \mathrm{Se}=\frac{T P}{T P+F N} \%(3) \\
& \mathrm{Sp}=\frac{T N}{F P+T N} \%(4)
\end{aligned}
$$

Where TP, TN, FP, and FN stand for true positive, true negative, false positive, and false negative respectively. For the sake of this calculations LI values were downgraded to a binary ranking variable: Right or Left. True positives were those cases in which the rank of the predictor (pair-wise LI) matched the Right or Left side on the task-related expressive and receptive fMRI activations. For this analysis, and giving the implications for clinical usage, patients were categorized as left lateralized either if their language LI (fMRI-based) were left (positive values) or having LI between -0.2 and +0.2 (usually accepted as bilateral representation of language). False negatives, for example, were cases where the LI from the predictor indicated right lateralization while language lateralization was actually left. Calculations were carried out for expressive and receptive modalities and reported in descriptive statistics.

To investigate possible interactions between our selected predictors and demographic and cognitive variables, the LI of predictors obtained at subject level were regressed against gender (binary rank), age, Global-IQ, Verbal-IQ, seizure onset and frequency.

All group comparisons and regressions were performed utilizing GNU-PSPP 0.7.9, 2012 (available at www.gnu.org/software/ pspp/pspp.html). Correlations $\mathrm{R}$ values greater than 0.3 will be accepted as significant.

\section{Results}

\section{Connectivity}

120 BA-pairs were possible per hemisphere, determined by the formula for the maximum number of unique connections in a mesh network with $\mathrm{N}$ nodes: $\mathrm{N}(\mathrm{N}-1) \div 2$. The divisor 2 is derived from the 
fact that a given pair will appear twice swapping origin and target. However, since this connectivity does not discriminate directionality, only one direction can be accepted. 109 pairs had at least one side with $\mathrm{Z}$ score over the threshold limit and were accepted for further analysis. A detailed demographics and the language scores in the sample are displayed in table 1 , while the connectivity results of the BA-pairs (PWC-LI and PWC-TDIF) are shown in table 2.

$\mathrm{T}$ test comparisons for GBAC is presented in table 3.

\begin{tabular}{|c|c|c|c|c|c|c|c|c|}
\hline \multicolumn{9}{|c|}{ Pairwise Brodmann Area Connectivity } \\
\hline \multicolumn{4}{|c|}{ Left } & \multicolumn{3}{|c|}{ Right } & \multirow{2}{*}{$\begin{array}{c}\text { LI } \\
\text { T Val } \\
\end{array}$} & \multirow{2}{*}{$\begin{array}{c}\text { PWC-T }_{\text {DIF }} \\
\text { Left - Right }\end{array}$} \\
\hline$\#$ & Seed - Target & $\mathbf{T}$ & P - FDR & Seed - Target & $\mathbf{T}$ & P - FDR & & \\
\hline 1 & 6L-9L & 9.45 & 0 & 6R-9R & 7.74 & 0 & 0.0995 & 1.71 \\
\hline 2 & $6 \mathrm{~L}-47 \mathrm{~L}$ & 9.07 & 0 & $6 \mathrm{R}-47 \mathrm{R}$ & 6.74 & 0 & 0.1474 & 2.33 \\
\hline 3 & $6 \mathrm{~L}-44 \mathrm{~L}$ & 6.93 & 0 & $6 \mathrm{R}-44 \mathrm{R}$ & 7.95 & 0 & -0.0685 & -1.02 \\
\hline 4 & $6 \mathrm{~L}-40 \mathrm{~L}$ & 6.04 & 0 & $6 \mathrm{R}-40 \mathrm{R}$ & 6.99 & 0 & -0.0729 & -0.95 \\
\hline 5 & 6L-39L & 5.75 & 0 & 6R-39R & 5.43 & 0 & 0.0286 & 0.32 \\
\hline 6 & $6 \mathrm{~L}-46 \mathrm{~L}$ & 5.36 & 0 & $6 \mathrm{R}-46 \mathrm{R}$ & 5.2 & 0 & 0.0152 & 0.16 \\
\hline 7 & $6 \mathrm{~L}-45 \mathrm{~L}$ & 5.25 & 0 & $6 \mathrm{R}-45 \mathrm{R}$ & 6.77 & 0 & -0.1265 & -1.52 \\
\hline 8 & $6 \mathrm{~L}-41 \mathrm{~L}$ & 5.02 & 0 & $6 \mathrm{R}-41 \mathrm{R}$ & 5.73 & 0 & -0.0660 & -0.71 \\
\hline 9 & $6 \mathrm{~L}-22 \mathrm{~L}$ & 4.69 & 0.0001 & $6 \mathrm{R}-22 \mathrm{R}$ & 5.96 & 0 & -0.1192 & -1.27 \\
\hline 10 & 6L-7L & 4.32 & 0.0002 & 6R-7R & 6.02 & 0 & -0.1644 & -1.70 \\
\hline 11 & 6L-38L & 4.23 & 0.0003 & 6R-38R & 3.26 & 0.0030 & 0.1295 & 0.97 \\
\hline 12 & $6 \mathrm{~L}-19 \mathrm{~L}$ & 4.05 & 0.0004 & 6R-19R & 2.79 & 0.0080 & 0.1842 & 1.26 \\
\hline 13 & $6 \mathrm{~L}-21 \mathrm{~L}$ & 4.03 & 0.0004 & 6R-21R & 3.41 & 0.0020 & 0.0833 & 0.62 \\
\hline 14 & 6L-37L & 3.12 & 0.004 & 6R-37R & 3.77 & 0 & -0.0943 & -0.65 \\
\hline 15 & $6 \mathrm{~L}-42 \mathrm{~L}$ & 2.17 & 0.0391 & $6 \mathrm{R}-42 \mathrm{R}$ & 3.24 & 0.0030 & -0.1978 & -1.07 \\
\hline 16 & $7 \mathrm{~L}-40 \mathrm{~L}$ & 8.43 & 0 & $7 \mathrm{R}-40 \mathrm{R}$ & 8.28 & 0 & 0.0090 & 0.15 \\
\hline 17 & 7L-19L & 7.66 & 0 & 7R-19R & 6.28 & 0 & 0.0990 & 1.38 \\
\hline 18 & 7L-37L & 6.92 & 0 & 7R-37R & 6.35 & 0 & 0.0430 & 0.57 \\
\hline 19 & 7L-9L & 6.5 & 0 & 7R-9R & 3.35 & 0.0040 & 0.3198 & 3.15 \\
\hline 20 & 7L-39L & 4.4 & 0.0003 & 7R-39R & 3.98 & 0.0010 & 0.0501 & 0.42 \\
\hline 21 & $7 \mathrm{~L}-47 \mathrm{~L}$ & 4.32 & 0.0003 & 7R-47R & 3.83 & 0.0010 & 0.0601 & 0.49 \\
\hline 22 & $7 \mathrm{~L}-41 \mathrm{~L}$ & 3.49 & 0.0026 & 7R-41R & 2.68 & 0.0170 & 0.1313 & 0.81 \\
\hline 23 & 7L-21L & 2.55 & 0.0227 & 7R-21R & 3.43 & 0.0040 & -0.1472 & -0.88 \\
\hline 24 & 7L-38L & 2.43 & 0.0286 & 7R-38R & 3.34 & 0.0040 & -0.1577 & -0.91 \\
\hline 25 & $7 \mathrm{~L}-45 \mathrm{~L}$ & 2.31 & 0.0358 & 7R-45R & 1.37 & & 0.2554 & 0.94 \\
\hline 26 & $7 \mathrm{~L}-42 \mathrm{~L}$ & 2.12 & 0.0492 & $7 \mathrm{R}-42 \mathrm{R}$ & 1.38 & & 0.2114 & 0.74 \\
\hline 27 & $7 \mathrm{~L}-46 \mathrm{~L}$ & 1.87 & & 7R-46R & 3.76 & 0.0020 & -0.3357 & -1.89 \\
\hline 28 & 7L-22L & 1.7 & & 7R-22R & 2.29 & 0.0340 & -0.1479 & -0.59 \\
\hline 29 & $9 \mathrm{~L}-47 \mathrm{~L}$ & 11.31 & 0 & 9R-47R & 7.12 & 0 & 0.2273 & 4.19 \\
\hline 30 & 9L-45L & 7.41 & 0 & 9R-45R & 5.38 & 0 & 0.1587 & 2.03 \\
\hline 31 & 9L-39L & 6.72 & 0 & 9R-39R & 5.58 & 0 & 0.0927 & 1.14 \\
\hline 32 & $9 \mathrm{~L}-22 \mathrm{~L}$ & 6.38 & 0 & 9R-22R & 3.67 & 0.0010 & 0.2697 & 2.71 \\
\hline 33 & 9L-19L & 6.35 & 0 & 9R-19R & 2.54 & 0.0190 & 0.4286 & 3.81 \\
\hline
\end{tabular}




\begin{tabular}{|c|c|c|c|c|c|c|c|c|}
\hline 34 & 9L-37L & 6.01 & 0 & 9R-37R & 4.3 & 0.0004 & 0.1659 & 1.71 \\
\hline 35 & $9 \mathrm{~L}-46 \mathrm{~L}$ & 5.97 & 0 & $9 R-46 R$ & 4.68 & 0.0004 & 0.1211 & 1.29 \\
\hline 36 & $9 \mathrm{~L}-40 \mathrm{~L}$ & 5.7 & 0 & $9 R-40 R$ & 5.22 & 0 & 0.0440 & 0.48 \\
\hline 37 & $9 \mathrm{~L}-44 \mathrm{~L}$ & 5.63 & 0 & 9R-44R & 4.92 & 0.0001 & 0.0673 & 0.71 \\
\hline 38 & 9L-21L & 5.61 & 0 & 9R-21R & 5.51 & 0 & 0.0090 & 0.10 \\
\hline 39 & 9L-38L & 5.27 & 0 & 9R-38R & 4.06 & 0.0004 & 0.1297 & 1.21 \\
\hline 40 & $9 \mathrm{~L}-41 \mathrm{~L}$ & 2.63 & 0.0136 & $9 R-41 R$ & 1.77 & & 0.1955 & 0.86 \\
\hline 41 & 19L-39L & 8.63 & 0 & 19R-39R & 7.33 & 0 & 0.0815 & 1.3 \\
\hline 42 & 19L-37L & 8.49 & 0 & 19R-37R & 9.26 & 0 & -0.0434 & -0.77 \\
\hline 43 & $19 \mathrm{~L}-47 \mathrm{~L}$ & 6.45 & 0 & 19R-47R & 4.56 & 0.0001 & 0.1717 & 1.89 \\
\hline 44 & $19 \mathrm{~L}-21 \mathrm{~L}$ & 5.14 & 0 & 19R-21R & 4.9 & 0.0001 & 0.0239 & 0.24 \\
\hline 45 & $19 \mathrm{~L}-46 \mathrm{~L}$ & 5.06 & 0 & 19R-46R & 4.49 & 0.0002 & 0.0597 & 0.57 \\
\hline 46 & 19L-38L & 4.23 & 0.0003 & 19R-38R & 4.94 & 0.0001 & -0.0774 & -0.71 \\
\hline 47 & $19 \mathrm{~L}-45 \mathrm{~L}$ & 4.06 & 0.0005 & 19R-45R & 2.51 & 0.0210 & 0.2359 & 1.55 \\
\hline 48 & $19 \mathrm{~L}-40 \mathrm{~L}$ & 2.59 & 0.0197 & 19R-40R & 1.83 & & 0.1719 & 0.76 \\
\hline 49 & $19 \mathrm{~L}-41 \mathrm{~L}$ & 2.5 & 0.0233 & 19R-41R & 2.9 & 0.0100 & -0.0741 & -0.4 \\
\hline 50 & $19 \mathrm{~L}-22 \mathrm{~L}$ & 2.43 & 0.0265 & 19R-22R & 3.71 & 0.0010 & -0.2085 & -1.28 \\
\hline 51 & 21L-39L & 10.12 & 0 & 21R-39R & 7.81 & 0 & 0.1288 & 2.31 \\
\hline 52 & $21 \mathrm{~L}-45 \mathrm{~L}$ & 9.06 & 0 & $21 \mathrm{R}-45 \mathrm{R}$ & 5.57 & 0 & 0.2386 & 3.49 \\
\hline 53 & $21 \mathrm{~L}-22 \mathrm{~L}$ & 8.67 & 0 & $21 \mathrm{R}-22 \mathrm{R}$ & 8.44 & 0 & 0.0134 & 0.23 \\
\hline 54 & $21 \mathrm{~L}-47 \mathrm{~L}$ & 8.45 & 0 & 21R-47R & 6.3 & 0 & 0.1458 & 2.15 \\
\hline 55 & 21L-38L & 7.33 & 0 & 21R-38R & 7.27 & 0 & 0.0041 & 0.06 \\
\hline 56 & 21L-37L & 6.74 & 0 & 21R-37R & 7.36 & 0 & -0.0440 & -0.62 \\
\hline 57 & $21 \mathrm{~L}-41 \mathrm{~L}$ & 5.07 & 0 & 21R-41R & 6.23 & 0 & -0.1027 & -1.16 \\
\hline 58 & $21 \mathrm{~L}-42 \mathrm{~L}$ & 4.84 & 0 & $21 \mathrm{R}-42 \mathrm{R}$ & 4.8 & 0 & 0.0041 & 0.04 \\
\hline 59 & $21 \mathrm{~L}-44 \mathrm{~L}$ & 2.75 & 0.0108 & $21 \mathrm{R}-44 \mathrm{R}$ & 2.28 & 0.0310 & 0.0934 & 0.47 \\
\hline 60 & $22 \mathrm{~L}-41 \mathrm{~L}$ & 9.86 & 0 & 22R-41R & 9.25 & 0 & 0.0319 & 0.61 \\
\hline 61 & $22 \mathrm{~L}-42 \mathrm{~L}$ & 9.83 & 0 & $22 \mathrm{R}-42 \mathrm{R}$ & 8.89 & 0 & 0.0502 & 0.94 \\
\hline 62 & $22 \mathrm{~L}-44 \mathrm{~L}$ & 7.17 & 0 & $22 \mathrm{R}-44 \mathrm{R}$ & 7.06 & 0 & 0.0077 & 0.11 \\
\hline 63 & $22 \mathrm{~L}-45 \mathrm{~L}$ & 7.14 & 0 & $22 \mathrm{R}-45 \mathrm{R}$ & 7.24 & 0 & -0.0070 & -0.1 \\
\hline 64 & $22 \mathrm{~L}-38 \mathrm{~L}$ & 6.89 & 0 & $22 \mathrm{R}-38 \mathrm{R}$ & 8.48 & 0 & -0.1034 & -1.59 \\
\hline 65 & $22 \mathrm{~L}-47 \mathrm{~L}$ & 6.48 & 0 & 22R-47R & 6.09 & 0 & 0.0310 & 0.39 \\
\hline 66 & $22 \mathrm{~L}-40 \mathrm{~L}$ & 5.71 & 0 & $22 \mathrm{R}-40 \mathrm{R}$ & 5.24 & 0 & 0.0429 & 0.47 \\
\hline 67 & $22 \mathrm{~L}-37 \mathrm{~L}$ & 4.43 & 0.0001 & 22R-37R & 6.87 & 0 & -0.2159 & -2.44 \\
\hline 68 & $22 \mathrm{~L}-39 \mathrm{~L}$ & 2.97 & 0.0063 & $22 \mathrm{R}-39 \mathrm{R}$ & 5.79 & 0 & -0.3219 & -2.82 \\
\hline 69 & $22 \mathrm{~L}-46 \mathrm{~L}$ & 1.08 & & $22 \mathrm{R}-46 \mathrm{R}$ & 3.51 & 0.0010 & -0.5294 & -2.43 \\
\hline 70 & $37 \mathrm{~L}-47 \mathrm{~L}$ & 6.56 & 0 & $37 \mathrm{R}-47 \mathrm{R}$ & 4.67 & 0.0001 & 0.1683 & 1.89 \\
\hline 71 & $37 \mathrm{~L}-40 \mathrm{~L}$ & 6.1 & 0 & $37 \mathrm{R}-40 \mathrm{R}$ & 4.64 & 0.0001 & 0.1359 & 1.46 \\
\hline 72 & 37L-45L & 5.27 & 0 & 37R-45R & 5.27 & 0 & 0.0000 & 0.00 \\
\hline 73 & $37 \mathrm{~L}-46 \mathrm{~L}$ & 4.92 & 0 & $37 \mathrm{R}-46 \mathrm{R}$ & 5.73 & 0 & -0.0761 & -0.81 \\
\hline 74 & 37L-38L & 4.66 & 0.0001 & $37 R-38 R$ & 5.17 & 0 & -0.0519 & -0.51 \\
\hline
\end{tabular}


Functional Connectivity as a Tool to Ascertain Language Localization in a Group of Children with Intractable Epilepsy

\begin{tabular}{|c|c|c|c|c|c|c|c|c|}
\hline 75 & $37 \mathrm{~L}-44 \mathrm{~L}$ & 4.34 & 0.0002 & $37 \mathrm{R}-44 \mathrm{R}$ & 3.83 & 0.0006 & 0.0624 & 0.51 \\
\hline 76 & 37L-41L & 4.06 & 0.0003 & 37R-41R & 4.15 & 0.0002 & -0.0110 & -0.09 \\
\hline 77 & 37L-39L & 3.66 & 0.0009 & 37R-39R & 5.05 & 0 & -0.1596 & -1.39 \\
\hline 78 & 37L-42L & 3.54 & 0.0012 & $37 \mathrm{R}-42 \mathrm{R}$ & 3.65 & 0.0009 & -0.0153 & -0.11 \\
\hline 79 & $38 \mathrm{~L}-47 \mathrm{~L}$ & 7.97 & 0 & $38 R-47 R$ & 6.28 & 0 & 0.1186 & 1.69 \\
\hline 80 & $38 \mathrm{~L}-42 \mathrm{~L}$ & 4.45 & 0.0002 & $38 R-42 R$ & 6.33 & 0 & -0.1744 & -1.88 \\
\hline 81 & $38 \mathrm{~L}-44 \mathrm{~L}$ & 4.15 & 0.0003 & $38 R-44 R$ & 2.9 & 0.0070 & 0.1773 & 1.25 \\
\hline 82 & $38 \mathrm{~L}-40 \mathrm{~L}$ & 3.84 & 0.0007 & 38R-40R & 2.81 & 0.0090 & 0.1549 & 1.03 \\
\hline 83 & 38L-39L & 3.77 & 0.0008 & 38R-39R & 4.5 & 0.0001 & -0.0883 & -0.73 \\
\hline 84 & $38 \mathrm{~L}-41 \mathrm{~L}$ & 3.75 & 0.0008 & 38R-41R & 6.12 & 0 & -0.2401 & -2.37 \\
\hline 85 & $38 \mathrm{~L}-45 \mathrm{~L}$ & 3.7 & 0.0009 & 38R-45R & 4 & 0.0004 & -0.0390 & -0.30 \\
\hline 86 & 39L-47L & 8.63 & 0 & 39R-47R & 6.26 & 0 & 0.1592 & 2.37 \\
\hline 87 & $39 \mathrm{~L}-45 \mathrm{~L}$ & 7.5 & 0 & 39R-45R & 3.6 & 0.0020 & 0.3514 & 3.90 \\
\hline 88 & $39 \mathrm{~L}-46 \mathrm{~L}$ & 3.09 & 0.006 & 39R-46R & 0.59 & & 0.6793 & 2.5 \\
\hline 89 & $39 \mathrm{~L}-42 \mathrm{~L}$ & -2.34 & 0.0339 & 39R-42R & 0.71 & & 0.5344 & -1.63 \\
\hline 90 & $40 \mathrm{~L}-41 \mathrm{~L}$ & 9.09 & 0 & 40R-41R & 6.97 & 0 & 0.1320 & 2.12 \\
\hline 91 & $40 \mathrm{~L}-42 \mathrm{~L}$ & 8.1 & 0 & 40R-42R & 5.24 & 0 & 0.2144 & 2.86 \\
\hline 92 & $40 \mathrm{~L}-47 \mathrm{~L}$ & 5.42 & 0 & 40R-47R & 6.08 & 0 & -0.0574 & -0.66 \\
\hline 93 & $40 \mathrm{~L}-46 \mathrm{~L}$ & 5.07 & 0 & 40R-46R & 6.75 & 0 & -0.1421 & -1.68 \\
\hline 94 & $40 \mathrm{~L}-44 \mathrm{~L}$ & 4.58 & 0.0001 & 40R-44R & 8.19 & 0 & -0.2827 & -3.61 \\
\hline 95 & $40 \mathrm{~L}-45 \mathrm{~L}$ & 2.26 & 0.0347 & $40 \mathrm{R}-45 \mathrm{R}$ & 4.18 & 0.0003 & -0.2981 & -1.92 \\
\hline 96 & $41 \mathrm{~L}-42 \mathrm{~L}$ & 9.07 & 0 & $41 \mathrm{R}-42 \mathrm{R}$ & 8.75 & 0 & 0.0180 & 0.32 \\
\hline 97 & $41 \mathrm{~L}-44 \mathrm{~L}$ & 5.11 & 0 & 41R-44R & 6.93 & 0 & -0.1512 & -1.82 \\
\hline 98 & 41L-47L & 3.54 & 0.002 & 41R-47R & 4.23 & 0.0003 & -0.0888 & -0.69 \\
\hline 99 & $41 \mathrm{~L}-45 \mathrm{~L}$ & 1.58 & & $41 \mathrm{R}-45 \mathrm{R}$ & 4.69 & 0.0001 & -0.4960 & -3.11 \\
\hline 100 & $42 \mathrm{~L}-44 \mathrm{~L}$ & 4.18 & 0.0004 & 42R-44R & 5.7 & 0 & -0.1538 & -1.52 \\
\hline 101 & $42 \mathrm{~L}-46 \mathrm{~L}$ & 2.35 & 0.0325 & $42 \mathrm{R}-46 \mathrm{R}$ & 0.86 & & 0.4642 & 1.49 \\
\hline 102 & $42 \mathrm{~L}-47 \mathrm{~L}$ & 2.27 & 0.0362 & 42R-47R & 2.62 & 0.0170 & -0.0716 & -0.35 \\
\hline 103 & $42 \mathrm{~L}-45 \mathrm{~L}$ & 1.83 & & 42R-45R & 3.37 & 0.0030 & -0.2962 & -1.54 \\
\hline 104 & $44 \mathrm{~L}-45 \mathrm{~L}$ & 8.82 & 0 & $44 \mathrm{R}-45 \mathrm{R}$ & 10.6 & 0 & -0.0917 & -1.78 \\
\hline 105 & $44 \mathrm{~L}-47 \mathrm{~L}$ & 7.37 & 0 & 44R-47R & 8.58 & 0 & -0.0759 & -1.21 \\
\hline 106 & $44 \mathrm{~L}-46 \mathrm{~L}$ & 5.52 & 0 & $44 \mathrm{R}-46 \mathrm{R}$ & 6.11 & 0 & -0.0507 & -0.59 \\
\hline 107 & $45 \mathrm{~L}-47 \mathrm{~L}$ & 10.15 & 0 & $45 R-47 R$ & 9.94 & 0 & 0.0105 & 0.21 \\
\hline 108 & $45 \mathrm{~L}-46 \mathrm{~L}$ & 7.09 & 0 & 45R-46R & 7.32 & 0 & -0.0160 & -0.23 \\
\hline 109 & $46 \mathrm{~L}-47 \mathrm{~L}$ & 5.48 & 0 & 46R-47R & 4.3 & 0.0003 & 0.1207 & 1.18 \\
\hline \multicolumn{9}{|c|}{$N=109$} \\
\hline Mean & & 5.36 & & & 5.15 & & 0.0207 & 0.1891 \\
\hline SD & & 2.43 & & & 2.11 & & 0.1883 & 1.5637 \\
\hline \multicolumn{2}{|c|}{ Mean+2SD } & & & & & & 0.3972 & 3.3165 \\
\hline
\end{tabular}

Table 2: BA pairwise connectivity results: Pairwise connectivity laterality Index (PWC-LI) and T difference (PWC-T ${ }_{D I F}$ ). Italic values indicate those connections that, although did not pass the threshold, were added to complete the pair. Bold values are those that passed the threshold of mean +2 SD. P-FDR: Probability of False Discovery Rate. Empty spaces represent not data available. Values less than 0.0001 have been considered as " 0 ”. 
There were no statistical differences between left and right GHC strength means, as a group (Mean left $=5.37$; Mean right $=5.15 ; \mathrm{p}=$ 0.57; CI (95\%): $-0.427<-0.176<0.779)$, see bottom of table 2 and 3 or by averaging lateralization indices either by BA pairs PWC-LI
(0.021) or by GBA-LI (0.0004). TDIF values derived from PWC-TDIF (0.189) or GBA-TDIF (0.253) analyses did not reveal any significant lateralization.

\begin{tabular}{|c|c|c|c|c|c|c|c|}
\hline \multirow{3}{*}{ BA } & \multicolumn{2}{|c|}{ Left Hemisphere } & \multicolumn{2}{|c|}{ Right Hemisphere } & \multirow{3}{*}{$\begin{array}{c}\text { P value } \\
\text { T-test } \\
\end{array}$} & \multirow{3}{*}{ GBA- LI } & \multirow{3}{*}{ GBA- $T_{\text {DIF }}$} \\
\hline & T Mean & SD & T Mean & SD & & & \\
\hline & & & & & & & \\
\hline 6 & 5.2987 & 1.9844 & 5.4000 & 1.7259 & 0.8826 & -0.0148 & -0.1013 \\
\hline 7 & 4.2157 & 2.2964 & 4.0243 & 2.0159 & 0.8100 & 0.0162 & 0.4555 \\
\hline 9 & 6.4957 & 1.9991 & 4.7029 & 1.6175 & 0.0119 & 0.1663 & 1.8646 \\
\hline 19 & 5.2031 & 2.1773 & 4.4646 & 2.1450 & 0.3736 & 0.0439 & 0.7385 \\
\hline 21 & 6.1815 & 2.4291 & 5.6392 & 1.8567 & 0.5286 & 0.0244 & 0.5423 \\
\hline 22 & 5.6953 & 2.7778 & 6.1660 & 2.1596 & 0.5900 & -0.1039 & -0.4707 \\
\hline 37 & 5.2547 & 1.5092 & 5.3380 & 1.5610 & 0.8830 & -0.0481 & -0.0833 \\
\hline 38 & 4.7621 & 1.5725 & 4.9614 & 1.7334 & 0.7526 & -0.0037 & -0.1993 \\
\hline 39 & 5.5377 & 3.4232 & 4.8923 & 2.2527 & 0.6400 & 0.1477 & 0.5225 \\
\hline 40 & 5.6100 & 2.0730 & 5.5708 & 1.9274 & 0.9606 & -0.0361 & 0.0392 \\
\hline 41 & 4.9823 & 2.6979 & 5.4154 & 2.2808 & 0.6400 & -0.1240 & -0.2100 \\
\hline 42 & 4.0315 & 3.3559 & 4.1631 & 2.8655 & 0.9152 & -0.0715 & 0.0160 \\
\hline 44 & 5.2831 & 1.9058 & 5.7331 & 3.0196 & 0.6536 & -0.0162 & -0.7083 \\
\hline 45 & 5.5320 & 2.8861 & 5.4540 & 2.5706 & 0.9382 & 0.0169 & -0.3057 \\
\hline 46 & 4.9362 & 2.6069 & 4.6477 & 2.1278 & 0.9640 & -0.0162 & 0.1809 \\
\hline 47 & 6.8980 & 2.4722 & 5.8400 & 1.8869 & 0.1836 & 0.0258 & 1.1586 \\
\hline Global & 5.3699 & 0.7467 & 5.1508 & 0.6213 & 0.5700 & 0.0004 & 0.2532 \\
\hline
\end{tabular}

Table 3: T test comparisons for Global Brodmann Area Connectivity (GBAC).

Significant asymmetry was found in the GBAC group comparisons of $\mathrm{T}$ means for BA9, being stronger on the left ( $\mathrm{p}=0.012$; CI $(95 \%): 0.495<1.793<3.09)$. The remaining $P$ values for the group differences ranged between to 0.183 (BA47) to 0.882 (BA6) (Table 3). The GBA-LI mean of BA9 was also the highest among BAs, but still below the standards utilized in clinical grounds (0.1663). BA9 was also the highest GBA-T ${ }_{D I F}(1.865)$ but below the significance threshold.

Significant statistical findings were noted on the pairwise related variables. The following pairs showed PWC exceeding 2 SD above the mean (Left: Mean $=5.36,+2 \mathrm{SD}=10.21$; Right: Mean = $5.18+2 \mathrm{SD}=9.40)$ : Left BA9-BA47 ( $\mathrm{T}=11.31)$; right BA44-BA45 $(\mathrm{T}$ $=10.6)$. PWC-LI ranged from -0.496 for pair BA41-BA45 to 0.679 for pair BA39-BA46. Six pairs showed PWC-LI exceeding the threshold of mean and 2SD (mean $=0.027 ;+2 \mathrm{SD}=0.397$ ): BA9-BA19 (0.429); BA22-BA46 (-0.529); BA39-BA46 (0.679); BA39-BA42 (0.534); BA41-BA45 (-0.496); and BA42-BA46 (0.464) (Figure 3). These values were designated as predictors. Only 2 pairs involved canonical core language areas BA22-BA46 and BA41-BA45.

Two BA explain 4 of the asymmetries: BA46 and BA39 as there is no differentiation based on directionality (Figure 4). Two areas had negative LI demonstrating right side lateralization.

PWC-T $_{\text {DIF }}$ ranged between -3.61, pair BA40-BA44, to 4.19, pair BA9-BA47. Threshold for significant variance was found at 3.36 


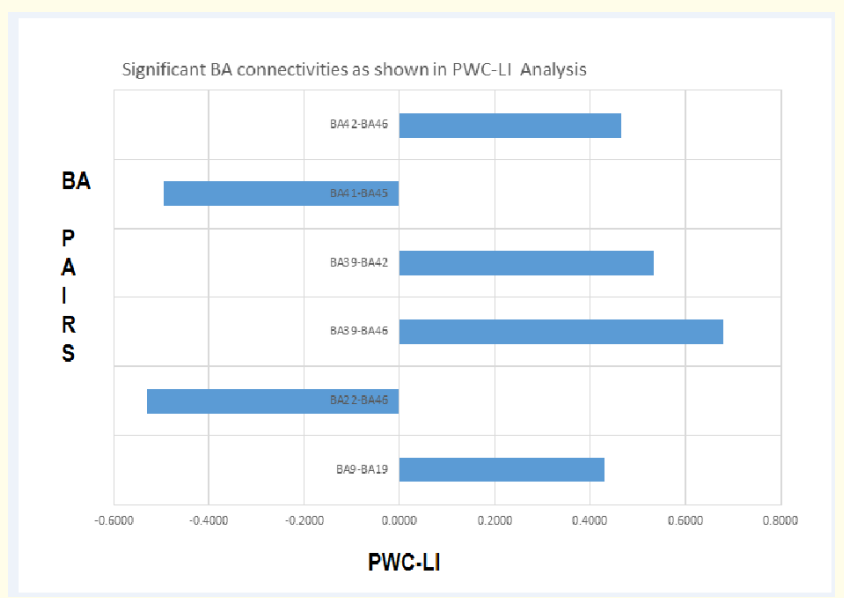

Figure 3: BA pairs exceeding the threshold of mean and 2SD after PWC-LI analysis.

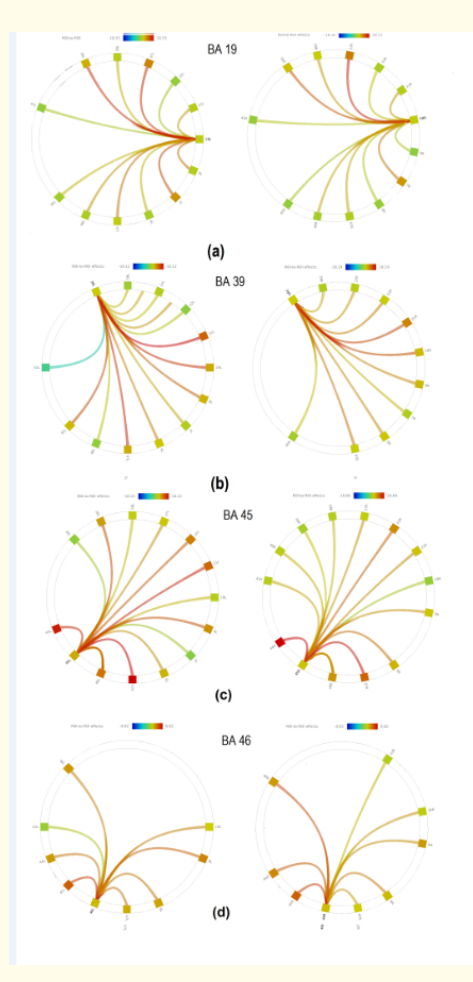

Figure 4: Illustration of asymmetries found per BA of interest. a) Connections found for BA 19; b) Connections found in BA 39;

c) Connections found on BA 45; d) Connections found in BA 46. Notice that 2 BA suffice to explain 4 of the asymmetries: BA46 and BA39, since there is no differentiation based on directionality.
$($ Mean $=0.250+2 \mathrm{SD}=3.108)$. Five pairs had statistical significant PWC-T $_{\text {DIF }}$ values: BA9-BA47 (4.19); BA9-BA19 (3.81); BA21-BA45 (3.49); BA39-BA45 (3.9); and BA40-BA44 (-3.61). See table 2.

Only three of these pairs involved the canonical core language areas: pair BA21-BA45, BA39-BA45 and BA40-BA44. The remaining significant differences were found between pairs formed with language ancillary areas.

\section{Correlations}

The regression values of the predictors against the language LI of all individual cases are presented in table 4 . No significant correlation was found between predictors and language LI. For expressive language, $\mathrm{R}$ values (Rexp) ranged between -0.136 for predictor BA22-BA46 and 0.246 for predictor BA45-BA41. For receptive language, $\mathrm{R}$ (Rrec) ranged between -0.109 for predictor BA22-BA46 and 0.128 for predictor BA39-BA46. Significant PWC-TDIF values regressed against patient's expressive language LI showed two significant R values 0.301 for pair BA39-BA45 and -0.428 for pair BA40-BA44. Correlations against patient's receptive language yield a significant correlation of $\mathrm{R}=0.419$ for predictor BA39-BA45. See table $4 \mathrm{a}$.

The sensitivity for language lateralization of the predictors from PWC-LI, and the PWC-T ${ }_{\text {DIF }}$-related pairs BA40-BA44 and BA39BA45 were analyzed at the subject level. The specificity analysis was dismissed as the denominator was 0 (true right carrying a result with infinite value) in many cases. PWC-LI related sensitivity for expressive and receptive language lateralization was above $80 \%$ only for predictor BA39-BA46 (80.56\%, for both domains). See table $4 \mathrm{a}$. Sensitivity of the PWC-T ${ }_{\mathrm{DIF}}$-significant pairs as predictors of expressive lateralization ranged from 25.7\% (BA40-BA44) to $71.4 \%$ (BA39-BA45), and for receptive language from $25.0 \%$ to $69.4 \%$, involving the same BA-pairs (Table 4b).

Regression of predictors against demographic and neuropsychology variables

The interaction between predictors and confounding variables gave the following results given in $\mathrm{R}$ ranges (Table 5): Predictor vs (1) age: -0.055 and 0.17 ; (2) gender: -0.333 and 0.117 ; (3) general IQ: -0.167 and 0.064; (4) verbal IQ: -0.105 and 0.247); (5) Seizure onset: -0.002 and 0.199 ; and (6) seizure frequency: 0.059 and -0.222 . The significant $\mathrm{R}$ of -0.333 accounted for a negative weak correlation between gender and PWC-LI of BA39-BA46, indicating that this pair is more left lateralized in girls (Table 4a). This 


\begin{tabular}{|c|c|c|c|c|c|c|}
\hline (a) & \multicolumn{6}{|c|}{ Regression coefficient R for BA PWC-LI (Predictors) } \\
\hline Effect of & $9-19$ & $22-46$ & $39-42$ & $39-46$ & $41-45$ & $42-46$ \\
\hline LI EXP & 0.1523 & -0.1359 & -0.0279 & -0.1013 & 0.2461 & -0.0517 \\
\hline LI REC & -0.0184 & -0.1085 & 0.1281 & 0.0655 & 0.0366 & -0.0216 \\
\hline Age & -0.0660 & 0.1797 & 0.0939 & -0.1779 & -0.0552 & 0.1130 \\
\hline VERB IQ & 0.2465 & 0.0063 & -0.1865 & 0.1883 & -0.1444 & -0.1053 \\
\hline GEN IQ & -0.0166 & -0.1672 & -0.1391 & 0.0639 & -0.0709 & -0.0704 \\
\hline Gender & -0.0491 & 0.0710 & -0.1241 & -0.3330 & 0.1171 & -0.0268 \\
\hline \multirow[t]{2}{*}{ (b) } & \multicolumn{6}{|c|}{ 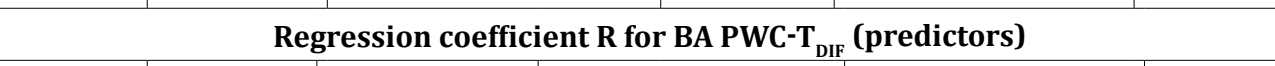 } \\
\hline & $9-47$ & $9-19$ & $21-45$ & -45 & $40-44$ & \\
\hline LI EXP & 0.1034 & 0.2681 & 0.2397 & 3011 & & \\
\hline LI REC & 0.2505 & 0.1872 & 0.2945 & 196 & & \\
\hline
\end{tabular}

Table 4: Correlation coefficient R for: (a) significant BA pairs by PWC-LI (Predictors) under effect of language lateralization indices, demographic and neuropsychological variables; (b) significant BA pairs by PWC- $\mathrm{T}_{\mathrm{DIF}}$

(Predictors) under effect of language lateralization indices.

Verb IQ: Verbal IQ; Gen IQ: General IQ; LI: Lateralization Index; Exp: Expressive Language; Rec: Receptive Language.

interaction was also validated grouping PWC-LI values by gender groups and analyzed with a T-test, case in which we obtained $\mathrm{P}=$ 0.047. No interaction of any other predictor was found in the same analysis ( $\mathrm{P}$ range between 0.47 and 0.87 ).

\section{Discussion}

This study assessed hemispheric, modular and pairwise asymmetries between canonical and ancillary language areas in a group of children with intractable partial epilepsy. We sought connectivity biomarkers that could predict language lateralization without requiring patient cooperation or invasive procedures. We hypothesize that (i) brain functional connectivity is asymmetric, (ii) LI derived from connectivity scalars correlates with language LI derived from fMRI, and (iii) that main asymmetries are found in canonical language areas.

We found new specific modular and pairwise asymmetries not previously described in children with epilepsy, but our first hypothesis was only partially validated as no global intra-hemispheric connectivity asymmetry was found. GBAC of BA9 was the only connectivity strength significantly asymmetric with leftward dominance. Of the 6-significant asymmetric PWC-LI, 4 were left and 2 were right-sided, but the asymmetry did not correlate to the language LI leading us to reconsider our second hypothesis.

Our third hypothesis (main findings related to core canonical language areas) was partially confirmed, as two pairs involved one of these areas - pairs BA41-BA45 and BA22-BA46 both exhibiting negative LIs. The remaining 4 pairs linked ancillary areas related to BA9, BA46 and BA39, and there were also no significant asymmetries for BA21 and BA44.

PWC-LI of BA39-BA46 was the only statistically significant predictor and also showed an acceptable performance in sensitivity, reaching $80 \%$ for both domains. With respect the PWC-TDIF, it is notable that only BA9-BA19 was also significant in the PWC-LI. The PWC-TDIF pair BA40-BA44 $\left(\mathrm{R}_{\exp }=-0.428\right)$ and BA39-BA45 $\left(\mathrm{R}_{\exp }=\right.$ $0.30, \mathrm{R}_{\text {rec }}=0.49$ ) showed statistically significant correlation with language LI. The sensitivity of the latter reached $69 \%$.

Our findings utilized a methodology similar to previous studies but also had important differences. Earlier studies utilized rsfMRI to assess connectivity between language areas. For example, the connectivity of expressive language regions along with the cingulate gyrus and posterior language areas have been studied 


\begin{tabular}{|c|c|c|c|c|c|c|c|}
\hline \multirow{2}{*}{\multicolumn{2}{|c|}{$\begin{array}{c}\text { (a) } \\
\text { Expressive Language }\end{array}$}} & \multicolumn{6}{|c|}{ Performance of PWBA-LI Connections (Predictors) } \\
\hline & & \multirow{2}{*}{$\begin{array}{c}9-19 \\
27 \\
\end{array}$} & \multirow{2}{*}{$\begin{array}{c}22-46 \\
19\end{array}$} & \multirow{2}{*}{$\begin{array}{c}39-42 \\
26\end{array}$} & \multirow{2}{*}{$\begin{array}{c}39-46 \\
29\end{array}$} & \multirow{2}{*}{$\begin{array}{c}41-45 \\
18\end{array}$} & \multirow{2}{*}{$\begin{array}{c}46-42 \\
24 \\
\end{array}$} \\
\hline True + & True Left & & & & & & \\
\hline False + & False Left & 0 & 0 & 0 & 0 & 0 & 0 \\
\hline True - & True Right & 1 & 1 & 1 & 0 & 0 & 0 \\
\hline False - & False Right & 8 & 16 & 9 & 7 & 18 & 12 \\
\hline \multicolumn{8}{|c|}{ Receptive Language } \\
\hline True + & True Left & 28 & 20 & 26 & 29 & 20 & 24 \\
\hline False + & False Left & 0 & 0 & 0 & 0 & 0 & 0 \\
\hline True - & True Right & 0 & 0 & 0 & 0 & 0 & 0 \\
\hline False - & False Right & 8 & 16 & 10 & 7 & 16 & 12 \\
\hline \multicolumn{8}{|c|}{ Expressive Language } \\
\hline Sensitivity & & inf & $54 \%$ & $74 \%$ & $81 \%$ & $50 \%$ & $67 \%$ \\
\hline Specificity & & $100 \%$ & inf & $100 \%$ & inf & $\inf$ & inf \\
\hline \multicolumn{8}{|c|}{ Receptive Language } \\
\hline \multicolumn{2}{|l|}{ Sensitivity } & $78 \%$ & $56 \%$ & $72 \%$ & $81 \%$ & $56 \%$ & $67 \%$ \\
\hline \multicolumn{2}{|l|}{ Specificity } & inf & inf & inf & inf & inf & inf \\
\hline \multicolumn{2}{|l|}{ (b) } & \multicolumn{6}{|c|}{ Performance of $\mathrm{T}_{\mathrm{DIF}}$-PWBA Connections (Predictors) } \\
\hline \multicolumn{2}{|c|}{ Expressive Language } & $9-47$ & 9 - 19 & $21-45$ & $39-45$ & $40-44$ & \\
\hline True + & True Left & 19 & 19 & 21 & 25 & 9 & \\
\hline False + & False Left & 1 & 0 & 0 & 0 & 0 & \\
\hline True - & True Right & 0 & 1 & 0 & 1 & 1 & \\
\hline False - & False Right & 16 & 16 & 15 & 10 & 26 & \\
\hline \multicolumn{8}{|c|}{ Receptive Language } \\
\hline True + & True Left & 20 & 19 & 21 & 25 & 9 & \\
\hline False + & False Left & 0 & 1 & 0 & 0 & 0 & \\
\hline True - & True Right & 0 & 0 & 0 & 0 & 0 & \\
\hline False - & False Right & 16 & 17 & 15 & 11 & 27 & \\
\hline \multicolumn{8}{|c|}{ Expressive Language } \\
\hline \multicolumn{2}{|l|}{ Sensitivity } & $54.29 \%$ & $54.29 \%$ & $58.33 \%$ & $71.43 \%$ & $25.71 \%$ & \\
\hline \multicolumn{2}{|l|}{ Specificity } & $0 \%$ & $100 \%$ & Inf & $100 \%$ & $100 \%$ & \\
\hline \multicolumn{8}{|c|}{ Receptive Language } \\
\hline Sensitivity & & $55.56 \%$ & $52.78 \%$ & $58.33 \%$ & $69.44 \%$ & $25.00 \%$ & \\
\hline Specificity & & Inf & 0 & Inf & Inf & Inf & \\
\hline
\end{tabular}

Table 5: Sensitivity and specificity for (a) BA- Pairwise LI based (PWC-LI) and (b) T difference pairwise based (PWC-T ${ }_{\text {DIF }}$ ) predictors.

in patients with temporal lobe epilepsy [20]. In this study fewer connections among language areas (clusters of language fMRI) were found in temporal lobe epilepsy (TLE) patients compared to healthy subjects, although no lateralization effect was found and there was no description of pairwise asymmetries. 
More recently, Doucet and colleagues compared 55 TLE patients with 23 healthy controls who underwent rs-FMRI [21]. Connectivity correlated with lateralization values (LI) from an expressive language task (verb-generation), and functional connectivity $\mathrm{T}$ values between the pars orbitalis and pars opercularis, and other brain areas. Interestingly, within the group of normal controls, a positive correlation with the language LI was found that involved connectivity between the right superior frontal cortex and the left SMA.

Our methodology correlated the signal time course from function-related ROIs rather than anatomical regions and included analyses of ancillary language areas. Despite these differences our findings provide additional support for the connectivity asymmetry of BA44 (pars opercularis of the inferior frontal gyrus) that we demonstrated with the $\mathrm{T}_{\mathrm{DIF}}$ method (BA40-BA44 PWC- $\mathrm{T}_{\mathrm{DIF}}=-3.61$ ).

Our findings are also concordant with a recent study [22], that utilized rs-fMRI to create complex correlation matrices for each hemisphere based on the Atlas of Intrinsic Connectivity of 192 homotopic areas described previously [23]. They utilized pairwise connectivity, while evaluating intra-hemispheric intrinsic connectivity asymmetry (HICA).

Despite these concordant findings, our methods differed substantially. Instead of ascertaining lateralization indices or contrasting left and right mean scalars, Joliot., et al. (2015) assessed hemispheric asymmetries by categorizing a matrix of the differences "between the left and right intra-hemispheric matrices of intrinsic correlation that were computed for each pair" as either typical or atypical, and correlated them to language lateralization indices obtained from standard fMRI.

Although we found strong asymmetry in pairs involving some of the canonical language areas, this was not unequivocal. Moreover, their LI values were not the strongest in the LI rank. For example, the left-most asymmetry for intra-core canonical language areas was 0.239 for BA21-BA45, whereas the overall left-most asymmetry was found for pair BA39-BA46 ( $\mathrm{LI}=0.679$ ). These findings emphasize the importance of left BA39, BA46 and BA9 since these 3 BA can explain 5 of the six most-significant pairs. It is striking that the most significant pairs associated with BA22 and BA45 (putative core language areas) formed with ancillary language areas rather than canonical pairs in other language domains. Likewise, it is difficult to understand the lack of connectivity asymmetry of all pairs related to BA21, and BA44 in the Pairwise caparisons (when $\mathrm{T}$ values were considered as absolute), or the right dominance of the BA22-BA46 connectivity.

The involvement of BA44 in language processing is well-known, producing activation across a wide range of fMRI language tasks (i.e. word generation, phonological and semantic fluency, metaphoric, syntactic and grammatical processing, lexical search, semantic memory retrieval, etc. see www.fmriconsulting.com/brodmann/ BA44.html for review). Ad-hoc data exploration revealed that some BA44-related pairs did not pass the thresholds. For example, pair BA44-BA39 had T values of 2.13 (left) and -0.52 (right), both below the passing threshold. LI scores with these values would yield a LI score of 0.60 , a score that was highly lateralized but not reported due to threshold constrains.

Another completely unexpected finding was the lack of asymmetry between BA21 pairs as BA21 is a core part of Wernicke's area. It is now established that a consistent structural connectivity asymmetry exists between receptive language areas (which includes BA21) and frontal expressive areas through the arcuate fasciculus $[24,25]$. Our results show very symmetric functional connectivity, with mean GBA-LI (BA21) $=0.034$ and maxima at pair BA21-BA45 (0.24).

The right-side lateralization of pairs BA22-BA46 and BA41BA45 was also unexpected. Left BA22 is the core of the receptive language area, along with BA21, but much less is known about the function of homotopic contralateral areas. A consensus is that right (or non-dominant) hemisphere has some advantage for processing prosody. Current understanding of how the cortex processes prosody is far less sophisticated than for declarative language making it difficult to speculate about the possible function of a functional connection between auditory secondary areas and areas of executive control (BA46).

A similar relationship exists for pair BA41-BA45, also with right side dominance. Ethofer utilized fMRI during recognition of prosodic expressions of 5 basic emotions [26]. Comprehension of affective prosody resulted in activation of BA22 and BA44, BA45 and BA47, the latter pair influencing executive control [27] and affective prosody [28].

The common finding of significance of BA9-BA19 in both methods to obtain a LI (absolute values vs. algebraic difference of $\mathrm{T}$

Citation: Byron Bernal., et al. "Functional Connectivity as a Tool to Ascertain Language Localization in a Group of Children with Intractable Epilepsy". Acta Scientific Neurology 4.4 (2021): 19-38. 
means) is of interest. The involvement of left BA9 in language has been previously established: It is involved in the activation of syntactic processing [29], verbal fluency [30], semantic categorization [31] and sentence generation [32]. Moreover, BA9 is a constitutive part of the largest cluster related to expressive functions [15] that includes left BA44, BA45, BA6, BA46, BA47 and anterior insula. However, the function of this connectivity is poorly understood. BA19 has been shown to be involved in processing the phonological properties of words by reading [33] and its utility in the reading and transfer of word decoding into expressive language is suggested.

Although the LIs are based on pair comparison of strength of activation, given by $\mathrm{T}$ absolute values, they may come from a negative value reflecting functional anti-correlation between the pair. One of the significant pairs was BA39-BA42. LI score for this pair was 0.534 , showing a clear left lateralization. However, the left $\mathrm{T}$ number is negative $(\mathrm{T}=-2.34)$ while the right is positive $(\mathrm{T}=0.71)$. The negatively correlated $\mathrm{T}$ values suggests an inhibitory input that involves de-activation. If this were the case, the left dominant PWC of BA39-BA42 would become a right dominant positive activation as reflected by PWC-TDIF which has a score of -1.63 .

The inhibitory effect of BA39 is reasonable as BA39 (the angular gyrus/inferior parietal lobule) is part of the default mode network, which has been found to have a negative correlation with task-related (i.e. salience and executive) networks [34,35]. However, the interplay between these two areas is poorly understood as BA42 is considered a primary auditory area. An association between BA41 and BA42 with complex phoneme perception with asymmetrical functions has been proposed [36].

\section{Limitations and Future Directions}

The age range of the present sample is limited as a forced consequence that this study is based in a pediatric neurological service. Moreover, the sample is biased to adolescents as the protocol required the performance of controlled tasks. Imaging data for children is far less common than for adults, therefore this study has a greater potential upside to understanding pediatric brain networks, particularly in patients with intractable epilepsy. The multiple disruptive effect epilepsy for brain connectivity has been already described. Functional connectivity is decreased between the default mode and executive control networks in patients with intractable left temporal lobe epilepsy [37]; connectivity changes are present and widespread during seizure free period in TLE [38]; changes are not only limited to cognitive complex networks but also to basic sensorimotor networks [39], at least in frontal lobe epilepsy and thalamo-cortical networks [40]. Many of our findings may therefore only be specific for certain types of epilepsy and could reasonably vary according to their heterogeneity and selectivity.

Our sample is unexpectedly unevenly distributed between males and females as we anticipated equal number of subjects by gender. Males constituted the majority of movement-related discarded studies. The superiority of female children in inhibitory motor task measures [41] likely explains this gender discrepancy. We found only a mild association between gender and lateralization related to BA39-BA46, indicating that this pair is more left lateralized in girls. Due to the small number of comparisons and the low association index we advise caution in assumptions derived from this finding.

The use of absolute values for the LI to keep the scalar as a ratio between -1 and 1 may obscure more significant differences. For instance, a pair having $\mathrm{T}$ values $-7.0 \mathrm{left}$, $7.0 \mathrm{right}$, will have the same LI than other pair having T values 7.0 left and 7.0 right. Although connectivity strength is the same, functional effects may be completely different, as negative values suggest inhibitory input, affecting the true lateralization as explained above. In an attempt to study this effect, the plain algebraic difference was computed as a scalar to regress against the language LI values. Remarkably, only the pair BA9-BA19 was represented in both approaches. However, this pair did not show important correlation with the language LI or neuropsychological scores.

A key end point of the study was the identification of a reliable biomarker of language asymmetry sufficiently sensitive to perform at the patient level. Notwithstanding, the maximum sensitivity obtained just reached $80 \%$ which seems insufficient to advise, counsel, encourage or dissuade brain surgery. Therefore, despite the complexity of the study and its stringent criteria to guarantee reliability of the results, our findings serve only as a point of departure for future studies.

The current findings are presented at an aggregated group level of epilepsy as we did not categorize patients by anatomical or pathological origin of the epilepsy and this variability may lead 
to conclusions that are not applicable to specific types of epilepsy. Therefore, the present results should be interpreted with caution, as they require replication and additional stratified studies.

An interesting limitation may be related to the functional MRI paradigm chosen for language mapping. In our experience of 20 years performing fMRI in children, the auditory description task is the most reliable, easily administered and well controlled paradigm at our disposal. However, when this task is contrasted with paradigms based on semantic decisions the activations differ slightly in terms of lateralization. The auditory description task yields more left lateralized activations for both expressive and receptive language than a task of synonyms/antonyms discrimination in a significant number of patients, suggesting that our tasks could have underestimated bilateral language representation. We did not explore this in the present study or any other correlation between connectivity LI and language LI based on another paradigm. Only patients with relatively good verbal skills perform enough well the semantic discrimination task. It is possible that a correlation with LI derived from this task would render different results.

\section{Conclusion}

In summary, using rs-fMRI data from a group of children with intractable partial epilepsy and functional connectivity between Brodmann areas involved in language processing, we have shown asymmetries that appear to be related to language lateralization. These asymmetries involve BA pairs BA9-BA19, BA22-BA46, BA39BA46, BA39-BA42, BA41-BA45 and BA42-BA46. In addition, pair BA40-BA44 shows significant left-right $\mathrm{T}$ differences. These significant pairs (predictors), however, have limited sensitivity to determine language lateralization ( $\max$ around $80 \%$ ).

Our findings do however suggest a possible disruptive effect of chronic focal epilepsy. These issues may be clarified by studies of normal patients and larger sample sizes. However, to the best of our knowledge, these results are new findings that further the understanding of language network connectivity in children with focal intractable epilepsy. We are confident that rs-fMRI procedures may soon provide a feasible method of probing language lateralization in pediatric patients requiring resective brain surgery.

\section{Ethical Publication Statement}

We confirm that we have read the Journal's position on issues involved in ethical publication and affirm that this report is consistent with those guidelines.

\section{Bibliography}

1. Lopez Gonzalez FJ., et al. "Drug-resistant epilepsy: definition and treatment alternatives". Neurologia 30.7 (2015): 439-446.

2. Jobst BC and Cascino GD. "Resective epilepsy surgery for drugresistant focal epilepsy: a review”. The Journal of the American Medical Association 313.3 (2015): 285-293.

3. Aneja S and Jain P. "Refractory epilepsy in children". Indian Journal of Pediatrics 81.10 (2014): 1063-1072.

4. Altman NR and Bernal B. "Brain activation in sedated children: auditory and visual functional MR imaging”. Radiology 221.1 (2001): 56-63.

5. Besseling RM., et al. "Aberrant functional connectivity between motor and language networks in rolandic epilepsy". Epilepsy Research 107.3 (2013): 253-262.

6. Carmon A., et al. "Asymmetries in hemispheric blood volume and cerebral dominance". Behavioral Biology 7.6 (1972): 853859.

7. Chiu HC and Damasio AR. "Human cerebral asymmetries evaluated by computed tomography". Journal of Neurology, Neurosurgery, and Psychiatry 43.10 (1980): 873-878.

8. Li X., et al. "Human torque is not present in chimpanzee brain". Neuroimage 165 (2018): 285-293.

9. Greicius MD., et al. "Persistent default-mode network connectivity during light sedation". Human Brain Mapping 29.7 (2008): 839-847.

10. Shimony JS., et al. "Resting-state spontaneous fluctuations in brain activity: a new paradigm for presurgical planning using fMRI". Academic Radiology 16.5 (2009): 578-583.

11. Ardila A., et al. "Connectivity of BA46 involvement in the executive control of language". Psicothema 28.1 (2016): 26-31.

12. Ardila A., et al. "How Localized are Language Brain Areas? A Review of Brodmann Areas Involvement in Oral Language”. Archives of Clinical Neuropsychology 31.1 (2016): 112-122.

13. Ardila A., et al. "How Extended Is Wernicke's Area? Meta-Analytic Connectivity Study of BA20 and Integrative Proposal". Journal of Neuroscience (2016): 4962562. 
14. Ardila A., et al. "Language and visual perception associations: meta-analytic connectivity modeling of Brodmann area 37". Behavioural Neurology (2015): 565871.

15. Bernal B., et al. "Broca's area network in language function: a pooling-data connectivity study". Frontiers in Psycholog 6 (2015): 687.

16. Rosselli M., et al. "[Angular gyrus connectivity model for language: a functional neuroimaging meta-analysis]". Revue Neurologique 60.11 (2015): 495-503.

17. Wechsler D. "Wechsler Adult Intelligence Scale-Fourth Edition (WAIS-IV) (2014).

18. Jenkinson M., et al. "FSL". Neuroimage 62.2 (2012): 782-790.

19. Whitfield-Gabrieli S and Nieto-Castanon A. "Conn: a functional connectivity toolbox for correlated and anticorrelated brain networks". Brain Connect 2.3 (2012): 125-141.

20. Waites AB., et al. "Functional connectivity networks are disrupted in left temporal lobe epilepsy". Annals of Neurology 59.2 (2006): 335-343.

21. Doucet GE., et al. "Resting-state functional connectivity predicts the strength of hemispheric lateralization for language processing in temporal lobe epilepsy and normal". Human Brain Mapping 36.1 (2015): 288-303.

22. Joliot M., et al. "Intra-hemispheric intrinsic connectivity asymmetry and its relationships with handedness and language Lateralization". Neuropsychologia 93 (2016): 437-447.

23. Joliot M., et al. "AICHA: An atlas of intrinsic connectivity of homotopic areas". Journal of Neuroscience Methods 254 (2015): 46-59.

24. Matsumoto R., et al. "Hemispheric asymmetry of the arcuate fasciculus: a preliminary diffusion tensor tractography study in patients with unilateral language dominance defined by Wada test". Journal of Neurology 255.11 (2008): 1703-1711.

25. Nucifora PG., et al. "Leftward asymmetry in relative fiber density of the arcuate fasciculus". Neuro Report 16.8 (2005): 791794.
26. Ethofer T., et al. "Cerebral pathways in processing of affective prosody: a dynamic causal modeling study". Neuroimage 30.2 (2006): 580-587.

27. Völlm B., et al. "Serotonergic modulation of neuronal responses to behavioural inhibition and reinforcing stimuli: an fMRI study in healthy volunteers". European Journal of Neuroscience 23.2 (2006): 552-560.

28. Del-Ben CM., et al. "The effect of citalopram pretreatment on neuronal responses to neuropsychological tasks in normal volunteers: an FMRI study". Neuropsychopharmacology is an International Scientific Journal and the Official Publication of the American College of Neuropsychopharmacology 30.9 (2005): 1724-1734.

29. Wang S., et al. "Broca's area plays a role in syntactic processing during Chinese reading comprehension". Neuropsychologia 46.5 (2008): 1371-1378.

30. Abrahams S., et al. "Functional magnetic resonance imaging of verbal fluency and confrontation naming using compressed image acquisition to permit overt responses". Human Brain Mapping 20.1 (2003): 29-40.

31. Hugdahl K., et al. "Left frontal activation during a semantic categorization task: an fMRI-study". International Journal of Neuroscience 99.1-4 (1999): 49-58.

32. Brown S., et al. "Music and language side by side in the brain: a PET study of the generation of melodies and sentences". European Journal of Neuroscience 23.10 (2006): 2791-2803.

33. Dietz NA., et al. "Phonological decoding involves left posterior fusiform gyrus". Human Brain Mapping 26.2 (2005): 81-93.

34. Aceko M., et al. "Is a Responsive Default Mode Network Required for Successful Working Memory Task Performance?" The Journal of Neuroscience 35.33 (2015): 11595-11605.

35. Sambataro F., et al. "Age-related alterations in default mode network: impact on working memory performance". Neurobiology of Aging 31.5 (2010): 839-852.

36. Bernal B and Ardila A. "From Hearing Sounds to Recognizing Phonemes: Primary Auditory Cortex is A Truly Perceptual Language Area". AIMS Neuroscience 3.4 (2016): 454-473. 
37. Zhang C., et al. "Characteristics of Resting-State Functional Connectivity in Intractable Unilateral Temporal Lobe Epilepsy Patients with Impaired Executive Control Function". Frontiers in Human Neuroscience 11 (2017): 609.

38. Lee H., et al. "Altered intrinsic functional connectivity in the latent period of epileptogenesis in a temporal lobe epilepsy model". Experimental Neurology 296 (2017): 89-98.

39. Woodward KE., et al. "Frontal lobe epilepsy alters functional connections within the brain's motor network: a resting-state fMRI study". Brain Connect 4.2 (2014): 91-99.

40. Peng SJ and Hsin YL. "Altered structural and functional thalamocortical networks in secondarily generalized extratemporal lobe seizures". Neuroimage Clinical 13 (2017): 55-61.

41. Mansouri FA., et al. "Sex dependency of inhibitory control functions". Biology of Sex Differences 7 (2016): 11.

\section{Assets from publication with us}

- Prompt Acknowledgement after receiving the article

- Thorough Double blinded peer review

- Rapid Publication

- Issue of Publication Certificate

- High visibility of your Published work

Website: www.actascientific.com/

Submit Article: www.actascientific.com/submission.php

Email us: editor@actascientific.com

Contact us: +919182824667 